\title{
Referral rates to specialists from general practice
}

\author{
A survey of demand for specialist restorative dental services

\section{P. J. Nixon and R. E. Benson Br Dent J 2005; 199: 161-163}

\section{Objectives}

To determine the level of demand from general dental practitioners (GDPs) for specialist restorative dental services in the Yorkshire region. To investigate barriers and promoters to referral and GDPs' perceptions of restorative mono-specialists.

Methods

A postal questionnaire was sent to 301 randomly selected GDPs (stratified for location) registered in the six Family Health Service Units in Yorkshire. Questionnaires were piloted prior to release; reminders were sent to non-respondents.

Results

A response rate of 72\% ( $n=217)$ was achieved; of these 195 questionnaires were useable (65\% useable response rate). Results showed a large demand for restorative specialist services. Main promoters for National Health Service (NHS) referral were dentolegal issues (77\% GDPs ranked this as a top 3 promoter) and for private referral increasing patient expectations (78\%). The top barriers to referral were length of waiting lists for NHS patients (79\% GDPs ranked this as a top 3 barrier) and high costs of treatment for private patients (88\%). Excessive distance to specialist centre was the greatest barrier common to both NHS and private referrals. Fifty-eight per cent of GDPs would prefer to refer private patients to mono-specialists, compared to $5 \%$ who would prefer restorative specialists.

\section{Conclusions}

There is a strong demand for specialist restorative services which may increase in the future. Results indicate a high regard for monospecialists. Overall demand is for a prompt, locally based, low cost referral service.

\section{IN BRIEF}

- A postal questionnaire investigating factors influencing specialist referral in restorative dentistry.

- Increasing patient expectations and the potential for medico-legal litigation are the main promoters of restorative referrals.

- Promoters and barriers to referral differ for NHS and private general dental practitioners.

- Overall demand is for a prompt, locally-based, cost effective specialist referral service.

\section{COMMENT}

This research is particularly timely. The recently published Standing Dental Advisory Committee (SDAC) document A review of the dentally based specialties and specialist lists ${ }^{1}$ states that: "Further work should be undertaken in relation to the future role and inter-relationships of restorative dentistry, the restorative mono-specialties and primary dental care practitioners." This suggests that the mono-specialties may not survive unless there is evidence of a demand for these services. Nixon and Benson's investigation of referral patterns within the Yorkshire Deanery is one of the first papers since the SDAC report to addresses some of these issues. From this work it is clear that general practitioners see value in referring to both restorative consultants and mono-specialists, with differences in preference depending upon whether referred from an NHS or private practitioner. The monospecialists in endodontics and periodontics are apparently held in high regard by private practitioners, but there is little demand for prosthodontists. This result is mirrored by the Department of Health's initiative in setting up working groups to determine the role of DwSpi's (Dentists with special interests) in the provision of endodontics and periodontics, but not prosthodontics, in the primary care setting.

A potential problem noted within the study is the large percentage of dentists who think they will refer future cases primarily for medicolegal reasons (75-87\%). Unless all consultant posts are replaced as they become empty, the number of restorative consultants in the UK is likely to reduce markedly in the next five years due to those reaching retirement age. If increasing numbers of patients are referred, concurrently, for medico-legal rather than purely clinical reasons there is a real risk that the system will be overburdened (with the current length of waiting lists already acting as a barrier to referral). This clearly exposes the myth that litigation improves patient care, as those with real clinical need will tend to be seen less quickly due to clogging of the system with 'defensive' referrals.

The paper recognises that patients' expectations are increasing. Most restorative consultants are aware of the amount of time taken to currently inform patients that their wishes, which may or may not be realistic, cannot be fulfilled by the NHS. Equally, many GDPs are aware that complex cases they have referred for treatment are often 'bounced' back to them for treatment that they may be unwilling to provide. The simpler, pragmatic, solutions that the consultant suggests do not always fully satisfy the patient's expectations but are often necessary due to workforce and financial stringencies. Is the solution an increased private provision? Apparently not, as this paper notes that cost is already a significant barrier to private referral.

This paper is an excellent analysis of the current situation in one Deanery. It would be valuable to extend the study to the rest of the UK and it would also be very interesting to repeat the study when Options for Change has been adopted.

C. C. Youngson, Hon Consultant in Restorative Dentistry, Liverpool University Dental Hospital, Pembroke Place, Liverpool doi: 10.1038/sj.bdj.4812606

1. Department of Health. http://www.advisorybodies.doh.gov.uk/sdac/specialistreview-report.pdf (accessed 20/12/04) 